\title{
Persistence of Paratropic Ring Currents in Nonplanar, Tub-Shaped Geometries of 1,3,5,7-Cyclooctatetraene
}

\author{
Remco W. A. Havenith, ${ }^{\dagger}$ Patrick W. Fowler, ${ }^{\ddagger}$ and Leonardus W. Jenneskens ${ }^{\star}, \S$ \\ Debye Institute, Theoretical Chemistry Group, Utrecht University, Padualaan 8, \\ 3584 CH Utrecht, The Netherlands, Department of Chemistry, University of Sheffield, \\ Sheffield, S3 7HF U.K., and Debye Institute, Organic Chemistry and Catalysis, \\ Utrecht University, Padualaan 8, 3584 CH Utrecht, The Netherlands
}

l.w.jenneskens@chem.uu.nl

Received November 22, 2005 (Revised Manuscript Received February 20, 2006 )

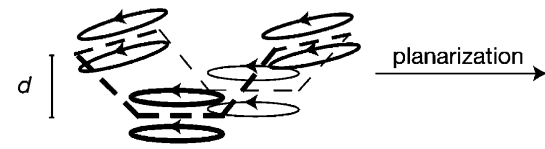

$0.76 \AA \geq d>0.62 \AA$

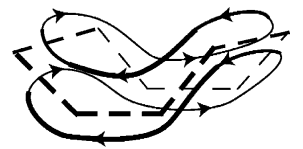

$0.62 \AA \geq d \geq 0.00 \AA$

Surprisingly, the anti-aromatic paratropic ring current of the planar $D_{4 h}$ transition state of 1,3,5,7-cyclooctatetraene (COT) survives almost all the way to the equilibrium tub-shaped $D_{2 d}$ COT structure (plane-to-plane distance, $d=0.76 \AA$ ); the current vanishes at ca. $80 \%$ of the geometric change and ca. $90 \%$ of the energy change from planar to tub geometry.

1,3,5,7-Cyclooctatetraene (COT) is a paradigm anti-aromatic $4 n \pi$ electron system. In an equilateral planar $D_{8 h}$ geometry, COT is predicted by simple Hückel theory to have a triplet ground state, but the real ground state has a closed-shell singlet electronic configuration and a nonplanar tub-shaped equilibrium geometry of $D_{2 d}$ symmetry. ${ }^{1}$ On the original definition of an anti-aromatic system as a "cyclic conjugated system [that] has its $\pi$ energy higher than that of a suitable reference compound, which is not cyclically delocalized", it is reasonable to suppose that the inherent anti-aromaticity of the $8 \pi$ electron cycle contributes to the nonplanarity of the equilibrium structure. However, calculations suggest that the $\pi$ resonance energy is negligible in planar conformations of $\mathrm{COT}^{3}$ and the larger $[4 n]$-annulenes ${ }^{4}$ indicating that the $\pi$ electrons have no role in driving COT to nonplanarity. An interpretation consistent with ideas of both strain release $e^{4,5}$

\footnotetext{
$\dagger$ Theoretical Chemistry Group, Utrecht University (affiliated with Organic Chemistry and Catalysis).

$\stackrel{+}{+}$ University of Sheffield.

$\S$ Organic Chemistry and Catalysis, Utrecht University.

(1) For recent reviews, see: (a) Klärner, F. G. Angew. Chem., Int. Ed. 2001, 40, 3977-3981; Angew. Chem. 2001, 113, 4099-4103. (b) Wiberg, K. B. Chem. Rev. 2001, 101, 1317-1331.

(2) Breslow, R.; Acc. Chem. Res. 1973, 6, 393-398.
}

and $\pi$-distortivity ${ }^{6}$ would be that the COT system moves from the equilateral $D_{8 h}$ to the bond-alternated $D_{4 h}$ structure by a first-order Jahn-Teller ( $\pi$-electronic) distortion and that further distortion to the out-of-plane $D_{2 d}$ geometry is driven primarily by release of angle strain, as $\mathrm{C}-\mathrm{C}-\mathrm{C}$ bond angles are thereby reduced from the $135^{\circ}$ angle of the planar octagon toward the ideal $\mathrm{sp}^{2}$ angle of $120^{\circ}$. From a combination of gas-phase electron-diffraction and single-crystal X-ray measurements, the $\mathrm{C}-\mathrm{C}-\mathrm{C}$ bond angle is $126.5^{\circ} 7$ (cf. RHF/6$31 \mathrm{G}^{* *}\left(\mathrm{~B} 3 \mathrm{LYP} / 6-31 \mathrm{G}^{* *}\right) 127.3^{\circ}{ }^{8}\left(127.7^{\circ}\right)$ ). Support for this view is given by the very small differences between the RHF/

(3) (a) Roth, W. R.; Lennartz, H.-W.; Vogel, E.; Leiendecker, M.; Oda, M. Chem. Ber. 1986, 119, 837-843. (b) Müllen, K.; Heinz, W.; Klärner, F. G.; Roth, W. R.; Kindermann, I.; Adamczek, O.; Wette, M.; Lex, J. Chem. Ber. 1990, 123, 2349-2371. (c) Karadakov, P. B.; Gerratt, J.; Cooper, D. L.; Raimondi, M. J. Phys. Chem. 1995, 99, 10186-10195.

(4) Wannere, C. S.; Moran, D.; Allinger, N. L.; Hess, B. A.; Schaad, L. J.; Schleyer, P. v. R. Org. Lett. 2003, 5, 2983-2986.

(5) Jones, M., Jr. Organic Chemistry, 2nd ed.; W. W. Norton \& Company: New York, 2000; Chapter 12.

(6) For a review, see: Shaik, S.; Shurki, A.; Danovich, D.; Hiberty, P. C. Chem. Rev. 2001, 101, 1501-1539.

(7) (a) Traettenberg, M. Acta Chem. Scand. 1966, 20, 1724-1726. (b) Claus, K. H.; Krüger, C. Acta Crystallogr. 1988, C44, 1632-1634.

(8) Fowler, P. W.; Havenith, R. W. A.; Jenneskens, L. W.; Soncini, A.; Steiner, E. Angew. Chem., Int. Ed. 2002, 41, 1558-1560. Angew. Chem. 2002, 114, 1628-1630. 
6-31G** optimized bond lengths ${ }^{8}$ in $D_{4 h}(\Delta R=0.153 \AA)$ and $D_{2 d}$ geometries $\left(\Delta R=0.155 \AA\right.$; expt. $\left.^{6,7} \Delta R=0.14 \AA\right)$ (For B3LYP/6-31G**, see Supporting Information, Table S24).

A clear distinction between the $D_{2 d}$ and $D_{4 h}$ structures is that in the $D_{4 h}$ geometry COT sustains a strong paratropic ring current, ${ }^{8}$ a signature of anti-aromaticity, ${ }^{9}$ which has disappeared in the tub-shaped equilibrium structure. The ipsocentric orbital model ${ }^{10}$ gives an explanation of currents in $[n]$-annulenes in terms of frontier-orbital topology according to which diatropic (aromatic) currents arise from unit change in angular momentum across the HOMO-LUMO gap, but paratropic (anti-aromatic) currents arise from the splitting of an angular momentum shell across the same gap. ${ }^{10 a}$ Since there is continuity in the orbital topologies along the $D_{2 d} \rightarrow D_{4 h}$ planarization coordinate, $d$, the plane-to-plane distance of the carbon ring (Figure 1), a paratropic ring

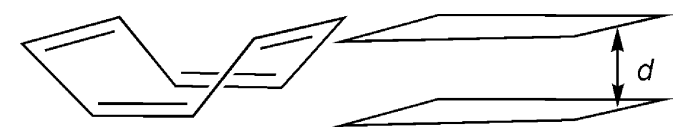

Figure 1. COT, showing schematically the definition of the planeto-plane distance, $d$.

current would be allowed by symmetry along the path. ${ }^{11}$ One probe of the extent to which $\pi$ electrons might be responsible for the nonplanarity of COT is the position of the onset of paratropic ring current. If current appears only very close to the planar $D_{4 h}$ geometry, requiring optimal $\mathrm{p}_{\pi}$ orbital overlap, then this would be consistent with a determining role for the $\pi$ electrons. If, however, the onset of current is close to the equilibrium geometry, then the $\pi$ electrons are spectators to a $\sigma$-driven process, in line with recent views on $\pi$-distortivity. ${ }^{6}$ This conclusion has consequences for synthetic work on clamped COTs. ${ }^{12}$ It has been shown that when COT is planarized by clamping with suitable saturated groups, such as bicyclo[2.1.1]hexeno, ${ }^{12 \mathrm{~d}}$ the central ring sustains the characteristic paratropic current of a $4 n \pi$ electron system. ${ }^{8}$ The present results suggest that this paratropic ring current could also be obtained in less-tightly clamped systems, ${ }^{12 \mathrm{e}}$ where COT has not been forced into a fully planar conformation (vide infra), and that the paratropic ring current may therefore be a more general feature of COT systems than has been supposed.

(9) Longuet-Higgins, H. C. Paramagnetic Ring Currents in the [4n]Annulenes in Aromaticity Special Publication No. 21; The Chemical Society, London, 1967.

(10) (a) Steiner, E.; Fowler, P. W. Chem. Commun. 2001, 2220-2221. (b) Steiner, E.; Fowler, P. W. J. Phys. Chem. A 2001, 105, 9553-9562. (c) Steiner, E.; Fowler, P. W.; Havenith, R. W. A. J. Phys. Chem. A 2002, 106, 7048-7056.

(11) Soncini, A.; Fowler, P. W.; Jenneskens, L. W. Phys Chem. Chem. Phys. 2004, 6, 277-284.

(12) (a) Kabuto, C.; Oda, M. Tetrahedron Lett. 1980, 21, 103-106. (b) Einstein, F. W. B.; Willis, A. C. J. Chem. Soc., Chem. Commun. 1981 526-528. (c) Ermer, O.; Klärner, F. G.; Wette, M. J. Am. Chem. Soc. 1986, 108, 4908-4911. (d) Matsuura, A.; Komatsu, K. J. Am. Chem. Soc. 2001, 123, 1768-1769. (e) Kiesewetter, M. K.; Reiter, R. C.; Stevenson, C. D. J. Am. Chem. Soc. 2005, 127, 1118-1119.
We report here a computational study of the development of the paratropic ring current in nonplanar geometries of COT. A suitable method for such a study is the ipsocentric ${ }^{10}$ treatment, which allows a decomposition of the results into physically nonredundant orbital contributions, using either canonical ${ }^{10 \mathrm{~b}}$ or localized molecular orbitals. ${ }^{10 \mathrm{c}}$ Current density was calculated for a series of $D_{2 d}$ geometries derived from tub-shaped COT by progressive reduction of $d$ from $0.76 \AA$ $\left(\mathrm{RHF} / 6-31 \mathrm{G}^{* *}\right)$ in the equilibrium tub geometry, by steps of $0.02 \AA$. At each step, all other structural parameters were reoptimized at the RHF/6-31G** (B3LYP/6-31G**) levels of theory using GAMESS-UK. ${ }^{13}$ At the limit of zero planeto-plane distance $d$, the RHF (B3LYP) geometries have $D_{4 h}$ symmetry and alternating bond lengths (see Supporting Information Tables S1a,b-S23a,b).

Maps of the current density induced in the $\pi$ system of COT by a magnetic field directed along the principal $C_{2}$ axis were computed at the ipsocentric CTOCD-DZ/6-31G**// RHF/6-31G** level using SYSMO.$^{14}$ Induced current density was calculated as the sum of the contributions from the four localized molecular orbitals ${ }^{15}$ that correlate with the four occupied $\pi$ orbitals in the $D_{4 h}$ planar limit. To follow the changes in current as the molecule is progressively flattened, maps were plotted for each plane-to-plane distance, $d$, in two projections: "horizontal" slices (a) in the median plane and (b) at a height of $1 a_{0}$ above the median plane. Figure 2 shows

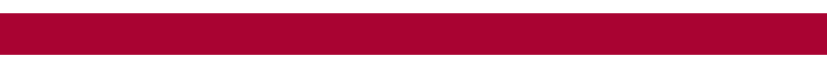

a)
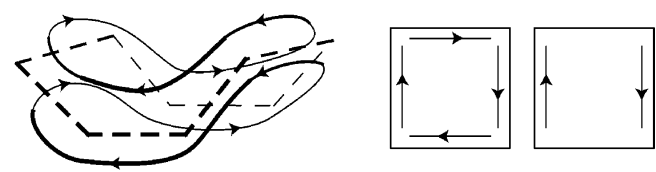

b)
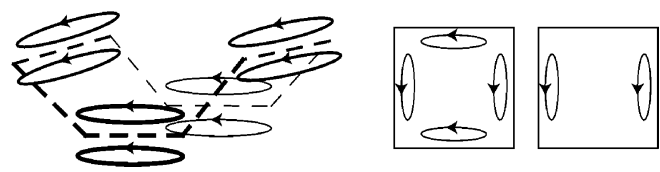

Figure 2. Signatures of (a) global paratropic (clockwise) and (b) localized diatropic (anticlockwise) currents in a typical nonplanar geometry of COT; in the median plane (left) and in the plane $1 a_{0}$ above the median plane (right). Global and localized currents can be distinguished by the different appearances of the two projected maps as represented schematically on the right.

schematically how global and local patterns can be recognized by their signature in the projected maps.

In Figure 3, the current density maps on the planarization pathway from the tub-shaped equilibrium structure $(d=0.76$

(13) Guest, M. F.; Bush, I. J.; van Dam, H. J. J.; Sherwood, P.; Thomas J. M. H.; van Lenthe, J. H.; Havenith, R. W. A.; Kendrick, J. Mol. Phys. 2005, 103, 719-747.

(14) Lazzeretti, P.; Zanasi, R. SYSMO Package; University of Modena: Modena, Italy, 1980, with additional routines by Steiner, E.; Fowler, P. W.; Havenith, R. W. A.; Soncini, A.

(15) Pipek, J.; Mezey. P. J. J. Chem. Phys. 1998, 90, 4916-4926. 

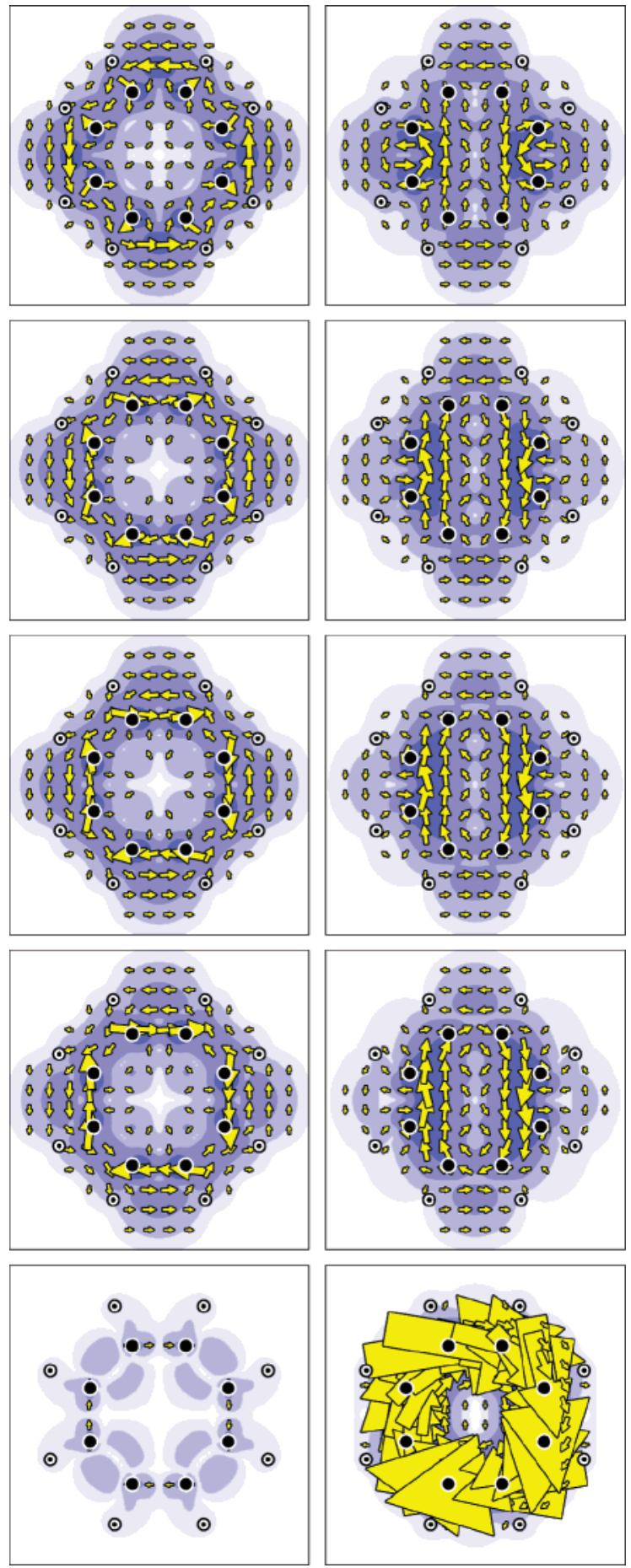

Figure 3. CTOCD-DZ/6-31G**//RHF/6-31G** $\pi$ current density maps for nonplanar $D_{2 d}$ COTs with $d=0.76,0.66,0.62,0.58$, and $0.05 \AA$ (from top to bottom) in two projections: (left column) in the median plane, (right column) at a height of $1 a_{0}$ above the median plane (see also Figures 1 and 2). The contours denote the modulus of the induced current density per unit magnetic field, and the vectors represent in-plane projections of this quantity.

$\AA)$, via intermediate structures $(d=0.66,0.62$, and $0.58 \AA$ ), to the planar $D_{4 h}$ limit ( $d=0.05 \AA$ ) are shown. The maps in the median plane present quite different appearances for the three structures (Figure 3, left column).
At the equilibrium structure, two of the formal double bonds lie $0.38 \AA$ above the median plane, and two $0.38 \AA$ below it, and the map therefore samples predominantly the outer lobes of four equivalent localized diatropic circulations; these appear as four sets of anticlockwise-directed arrows. In the planar limit, the $\pi$ orbitals have zero amplitude in the median plane, and the map for the structure with $d=0.05$ $\AA$ is correspondingly devoid of significant $\pi$ current.

The maps in the $1 a_{0}$ plane again show qualitative differences in signature (cf. also Figure 2) between the three structures (Figure 3, right). For the equilibrium structure, two of the formal double bonds now lie much closer to the plotting plane, and the map is dominated by the inner lobes of their localized diatropic circulations, which appear as two sets of clockwise-directed arrows in the map. Near the planar limit, the $1 a_{0}$ map shows the strong paratropic $\pi$ current of the COT ring. ${ }^{8}$ The very large arrows indicate the high strength of the current at exact planarity-a consequence in ipsocentric theory of its origin in the Jahn-Teller splitting of an originally degenerate level.

From Figure 3, it is clear that the crossover point between the two regimes, from localized current in the tub structure to global ring current in the planar structure, occurs near $d$ $=0.62 \AA$. At this point, the map in the median plane exhibits the transition from outer anticlockwise to inner clockwise circulation. At the same time, in the $1 a_{0}$ map the two islands of current are joining to make a single global paratropic circulation.

Why, on the route from $D_{2 d}$ tub to $D_{4 h}$ planar geometry, should the current switch on in structures that are far from planar? The profile of relative energy, $\Delta E\left(\mathrm{RHF} / 6-31 \mathrm{G}^{* *}\right)$, along the planarization coordinate is shown in Figure 4a,
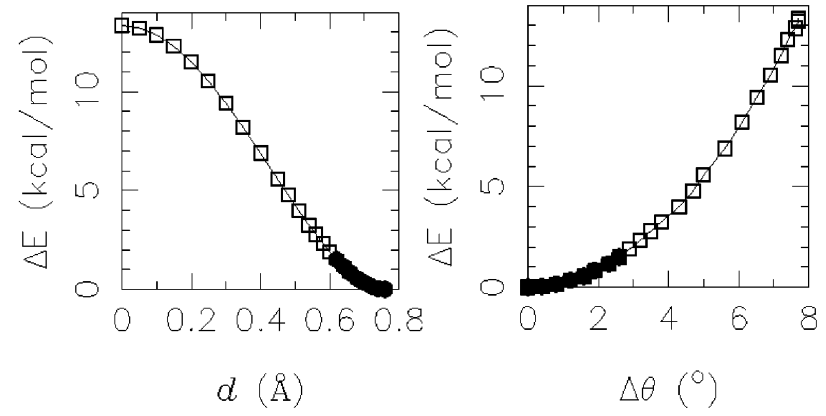

Figure 4. Variation of relative energy $\left(\Delta E\left(\mathrm{RHF} / 6-31 \mathrm{G}^{* *}\right)\right.$ in $\mathrm{kcal} /$ mol) of (constrained) $D_{2 d}$ and planar $\left(D_{4 h}\right)$ forms of COT with (a) plane-to-plane distance $d$ and (b) change in $\mathrm{C}-\mathrm{C}-\mathrm{C}$ bond angles $(\Delta \theta)$ along the planarization pathway following $\Delta E \approx \Delta \theta^{n}$ with $n$ $=1.9733,{ }^{17}$ in which $d$ is constrained and all other structural parameters are optimized [while the (constrained) $D_{2 d}$ COTs with $0.76 \AA \geq d>0.62 \AA$ (四) do not sustain a paratropic ring current, those with $0.62 \AA \geq d>0.00 \AA(\square)$ do].

where it is seen that the $d$ value of $0.62 \AA$ is energetically close to the equilibrium structure $\left(\Delta E\left(\mathrm{RHF} / 6-31 \mathrm{G}^{* *}\right)=1.50\right.$ $\mathrm{kcal} / \mathrm{mol}$; see Supporting Information Table S24) ${ }^{16}$ Replotting the relative energy, $\Delta E\left(\mathrm{RHF} / 6-31 \mathrm{G}^{* *}\right)$, as a function 
of the change of the $\mathrm{C}-\mathrm{C}-\mathrm{C}$ angle $(\Delta \theta$, Figure $4 \mathrm{~b}$, Supporting Information Table S24) shows the quasi-parabolic dependence $\left(\Delta E(\mathrm{RHF} / 6-31 \mathrm{G} * *) \approx \Delta \theta^{n}\right.$ with $n=1.9733,{ }^{17}$ Supporting Information Figures $\mathrm{S} 1-\mathrm{S} 2$ ) that would be expected if the major contributor to stabilization of the $D_{2 d}$ tub geometry is release of angle strain in the $\sigma$-skeleton.

Analysis of the (constrained) structures using the $\pi$-orbital axis vector (POAV) formalism ${ }^{18}$ shows that rehybridization at the carbon centers is a small effect: the POAV angles change from $93.2^{\circ}$ in the equilibrium structure to $90.0^{\circ}$ in the planar structure, that is, the orbitals contributing to the $\pi$ system are essentially pure $\mathrm{p}_{\pi}$. Therefore, the ring current will be proportional to their overlap.

Since the changes in bond length along the planarization coordinate are small (see Supporting Information Table S24), the major factor influencing the paratropic ring current is the angular mismatch between $\mathrm{p}_{\pi}$ orbitals across the longer $\mathrm{C}-\mathrm{C}$ bond. This misalignment varies from ca. $50^{\circ}$ (equilibrium) to $0^{\circ}$ (planar). In the region of $d$, where paratropic current first appears, the misalignment angle has fallen to $40-45^{\circ}$.

Starting from the $D_{4 h}$ planar geometry of COT, the energy gain on closing of the $\mathrm{C}-\mathrm{C}-\mathrm{C}$ bond angle drives the outof-plane distortion, which is accompanied by an increased misalignment of $\mathrm{p}_{\pi}$ orbitals, which in turn reduces and eventually switches off the paratropic current. Misalignments of $40-45^{\circ}$ indicate reduced overlap integrals (range $0.707-$ 0.766, see Supporting Information Table S24) and are associated with loss of delocalization in $\pi$-conjugated systems. ${ }^{18}$

It is striking that the paratropic ring current survives almost all the way from the planar COT to its equilibrium structure. In the tub-shaped equilibrium geometry, COT has lost its paratropic ring current, but a minor distortion, entailing little

(16) The RHF/6-31G** (B3LYP/6-31G**) energy difference between equilibrium (tub-shaped) $D_{2 d}$ and planar $D_{4 h}$ COT is $13.33(9.88) \mathrm{kcal} / \mathrm{mol}$ (Supporting Information, Table S24). The CCSD(T)/cc-pVTZ energy difference equals $12.74 \mathrm{kcal} / \mathrm{mol}$.

(17) RHF/6-31G** (B3LYP/6-31G**) $\Delta E \approx \Delta \theta^{n}$ was fitted by linear regression of $\ln (\Delta E)=n \ln (\Delta \theta)+\mathrm{C}^{\prime}$, which gave $n=1.9733$ (1.9582) and $\mathrm{C}^{\prime}=-1.4654(-1.6249)$ with $r^{2}=0.9996$ (0.9997) (Supporting Information Figure S1 (Figure S2)).

(18) Haddon, R. C. J. Am. Chem. Soc. 1987, 109, 1676-1685. increase in energy, would be sufficient to restore it. Indeed, the ${ }^{1} \mathrm{H}$ NMR spectra of the recently synthesized nonplanar cycloprop[8]annulene ${ }^{12 \mathrm{e}}$ support this view; CTOCD-DZ/6$31 \mathrm{G} * * / / \mathrm{RHF} / 6-31 \mathrm{G} * *$ calculations show that this molecule sustains a paratropic ring current (Figure 5).
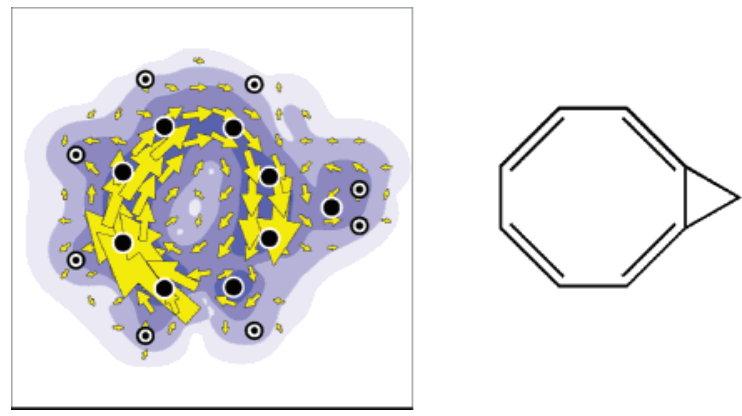

Figure 5. CTOCD-DZ/6-31G**//RHF/6-31G** $\pi$ map of cycloprop[8]annulene ${ }^{12 \mathrm{e}}$ plotted at $1 a_{0}$ above the median plane. The RHF/ 6-31G** optimized geometry has $C_{2}$ symmetry with the axis shown horizontal. The arrows show a strong paratropic ring current in the nonplanar COT cycle. The median plane lies at an angle to the plotting plane, causing the upper half of the current to be emphasized relative to the lower half in this projection.

Acknowledgment. The Royal Society/Wolfson Scheme for a Research Merit Award (P.W.F.). The Netherlands Organisation for Scientific Research (NWO, Grant 700.53.401, R.W.A.H., and NWO/NCF, project number SG-032).

Supporting Information Available: RHF/6-31G** (B3LYP/6-31G**) and $\pi$-orbital axis vector data (Tables S1a,b-S23a,b and S24) and Figures S1-S2. This material is available free of charge via the Internet http://pubs.acs.org.

OL052822C 